$$
\begin{aligned}
& \text { تأثير عوامل المناخ الداخلي في بعض المؤشرات الإنتاجية للاجاج البياض إبراض }
\end{aligned}
$$

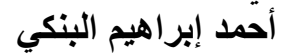

$$
\begin{aligned}
& \text { قسم الإنتاج الحيواني ـ كلية الزراعة ـ جامعة الفرات ـ سوريا }
\end{aligned}
$$

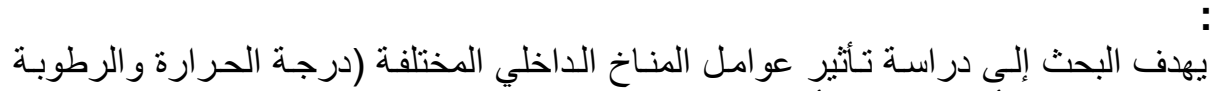

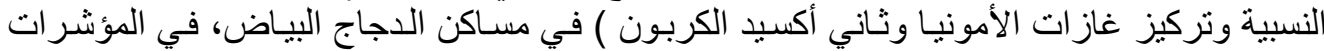

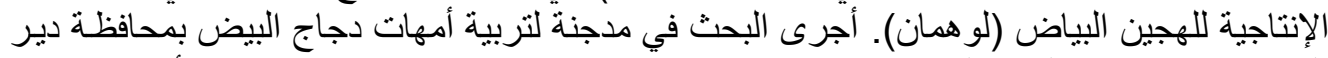

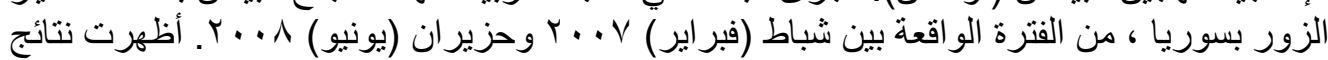

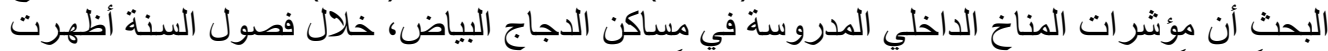

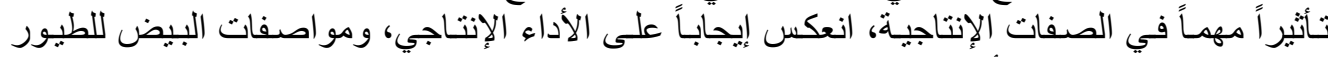

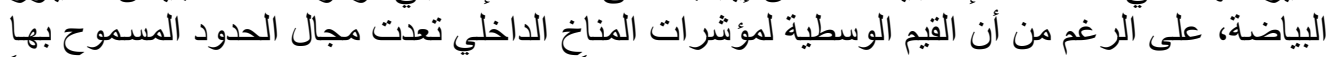

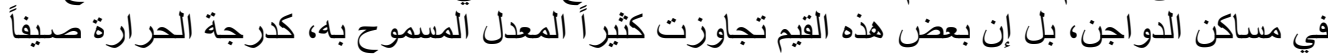

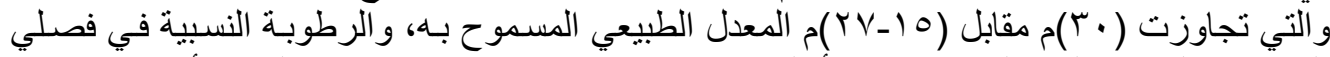

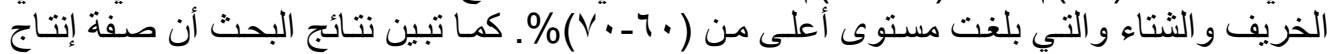

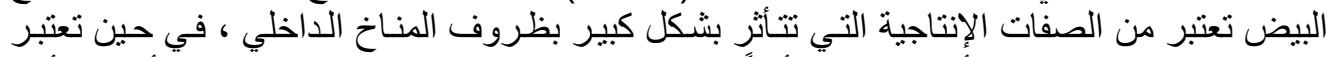

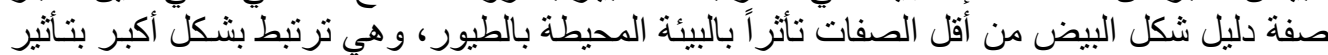

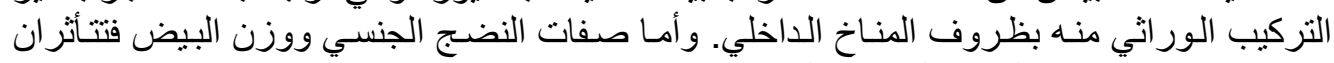
بوجود نر ابط قوبي بين البيئة و التركيب الور الوراثي.

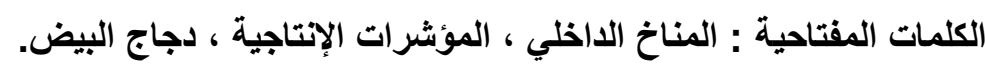

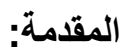

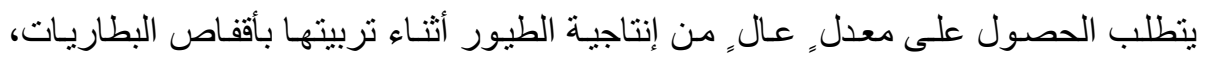

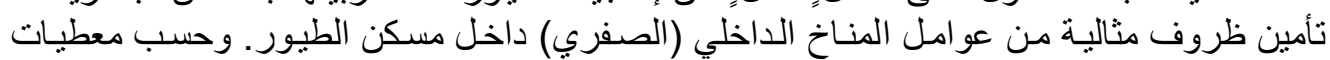

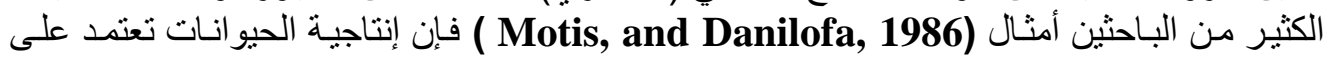

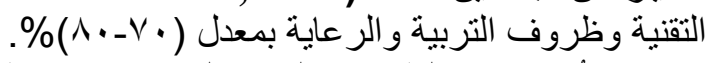

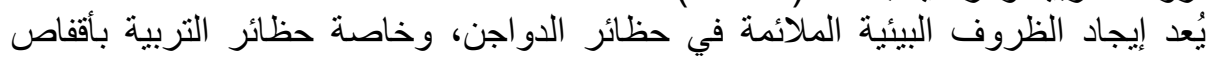

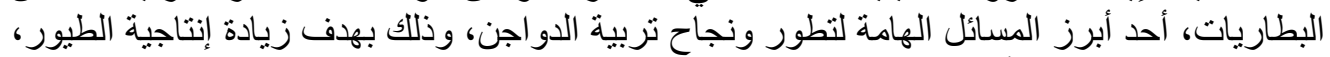

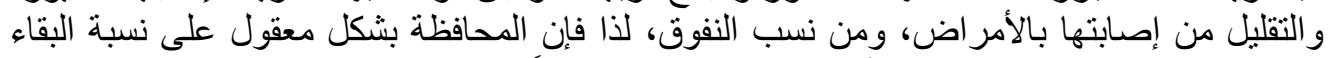

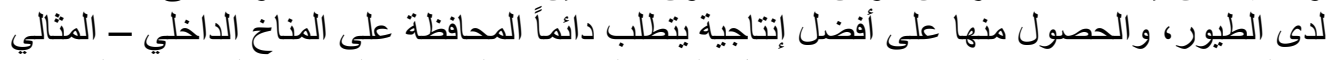

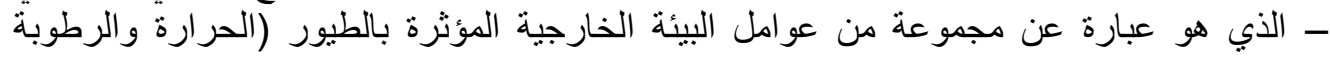

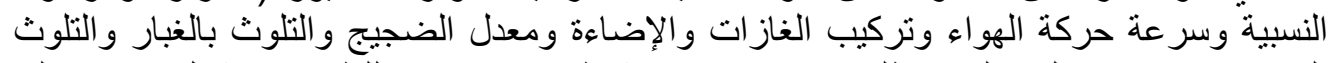

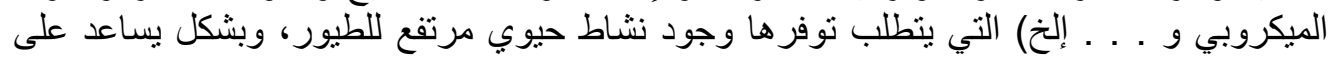

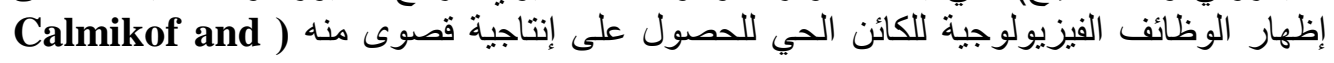

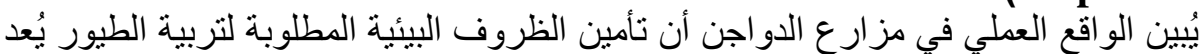

.(Alepastrof, 1975

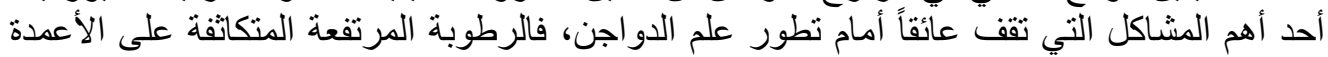

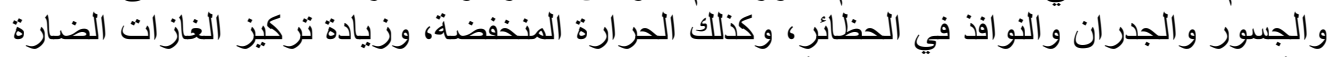

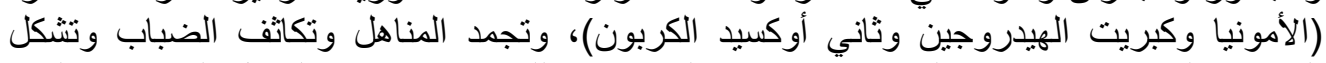

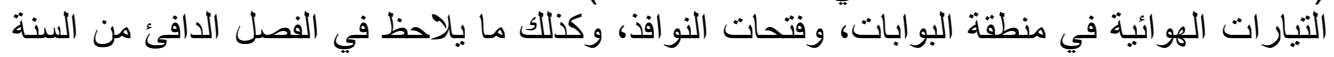

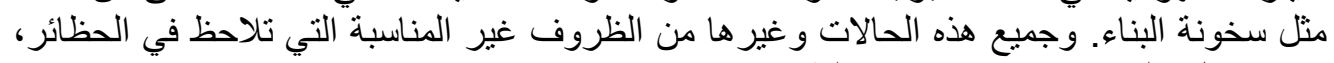

تؤثر بشكل سلبي في صحة و إنتاجية الطيور (Dutt, 1998).

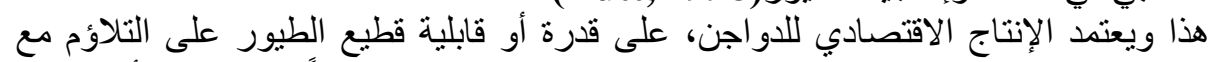

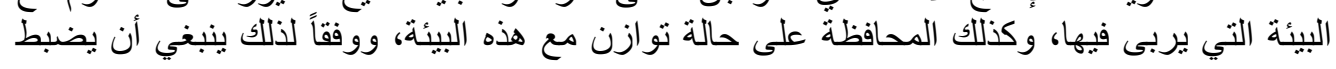

Fayoum J. Agric. Res. \& Dev., Vol.23, No.2, (B) July, 2009 
المناخ الداخلي (الصغري) لتلبية منطلبات واحتياجات نوع الطيور المرباة، والزمرة العمرية لها، وأغراض الإنتاج بأقل تكلفة ممكنة. (الغنة )

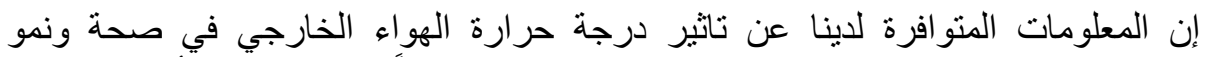

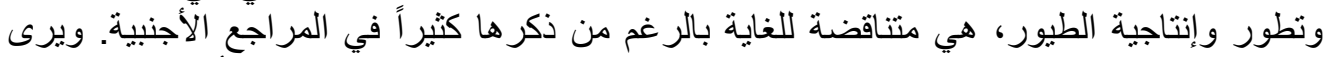

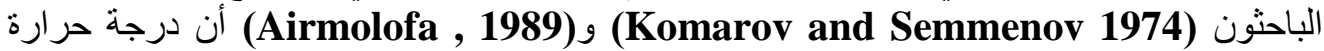

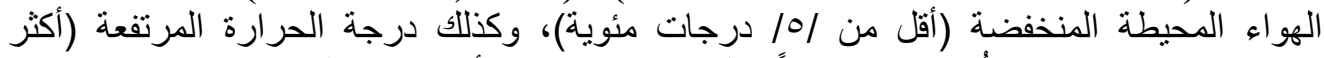

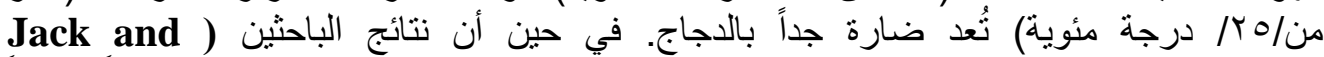

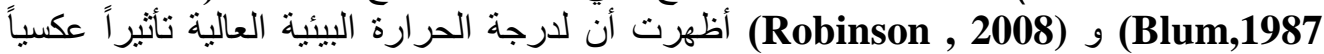

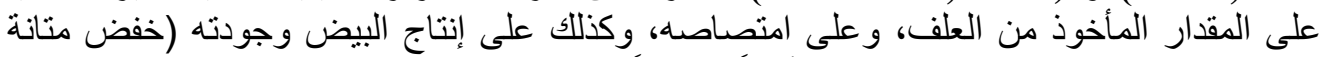

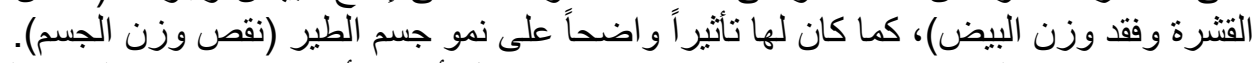

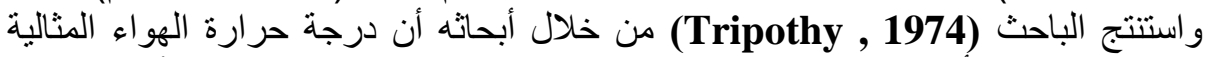

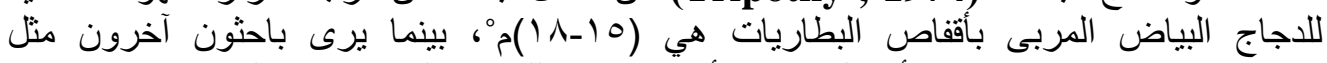
أن الحرارة الأكثر ملائمة للاجاج البياض هي الدرجة (Petersen, et al, 1976) و وأما الباحث (Moul and Katle,1990) فيشير من خلال أحد أعماله أنه يوجد لكل أنواع أنداع وحنى (r)

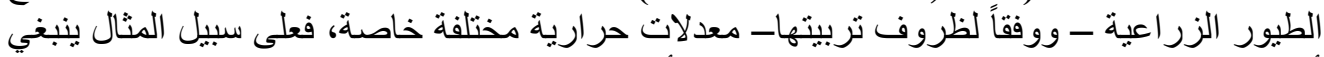

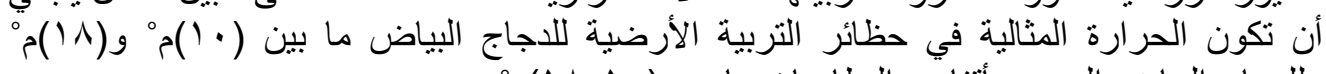

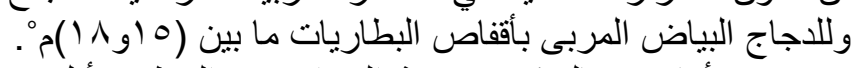

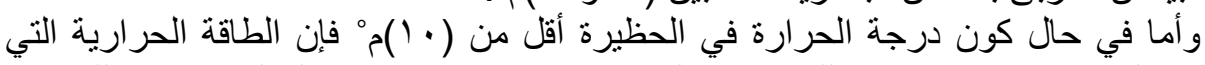

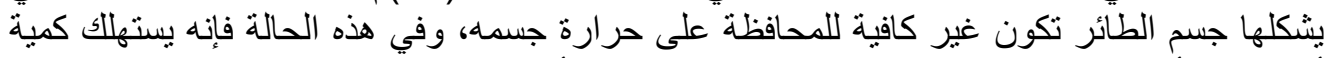

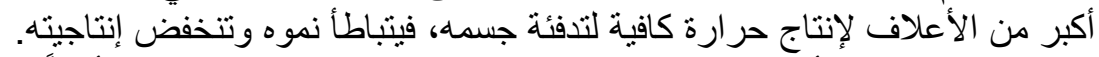

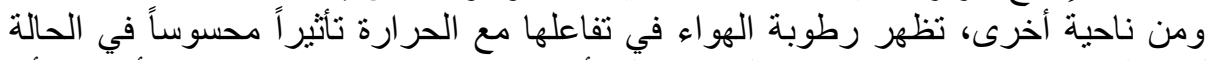

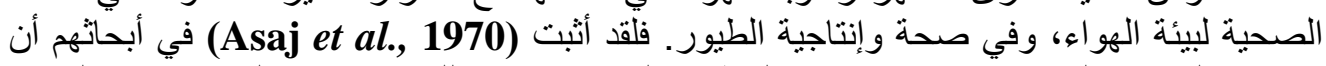

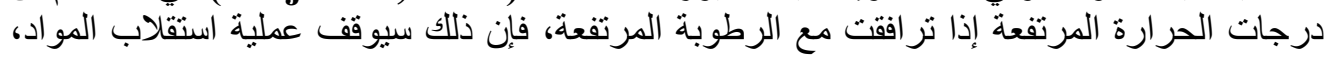

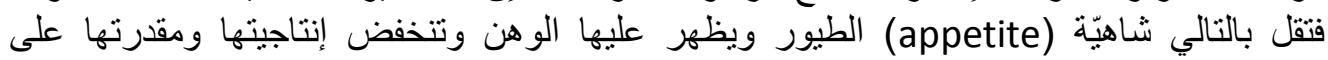
مقاومة الأمر اض المعدية وغير المعالة المعية.

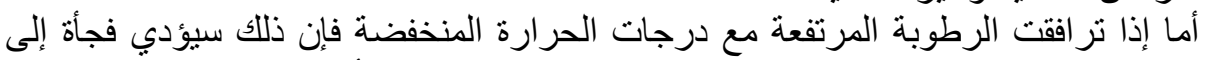

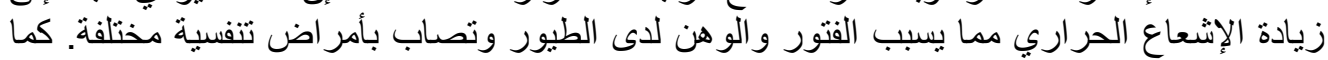

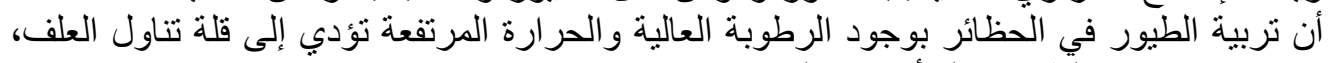
و انخفاض إنتاجية الطيور وقئة الطئة أوز انها الحية.

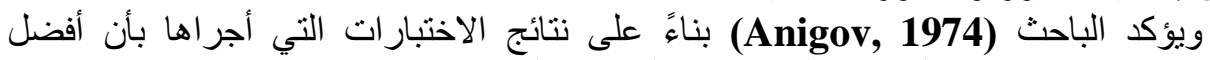

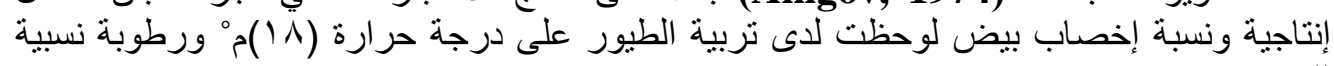
في حين أن الباحث (Arora and Karla, 1992) فيرى بأن الرطوبة النسبية الأكثر

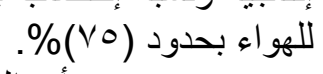

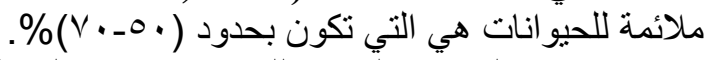

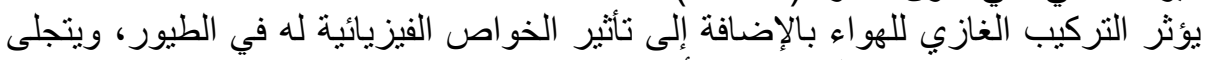

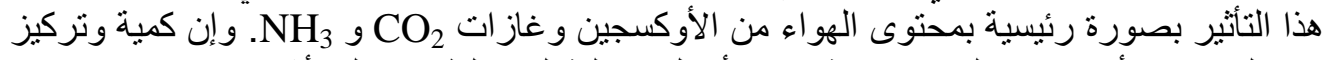

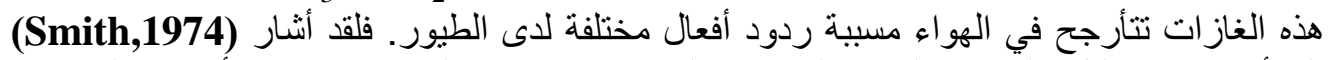

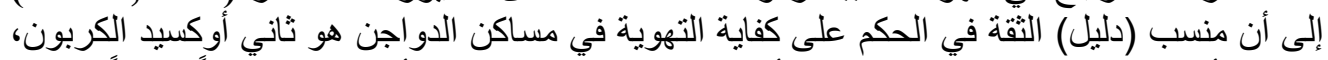

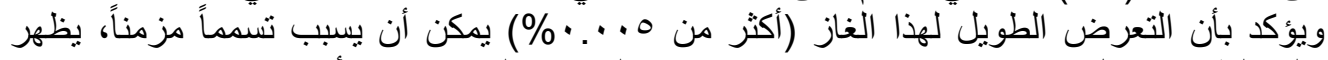

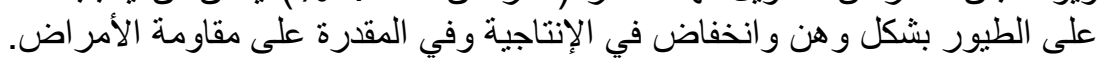

Fayoum J. Agric. Res. \& Dev., Vol.23, No.2, (B) July, 2009 
وفي السنة ذاتها قدم كل من (Komarov and Semmenov 1974) توصية بأن الحد المسموح به من غاز

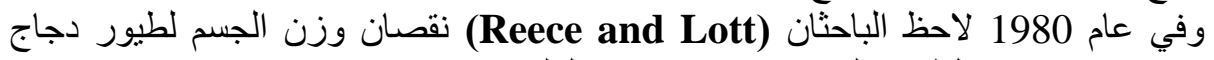

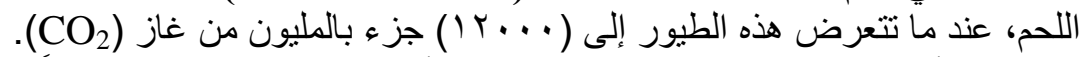

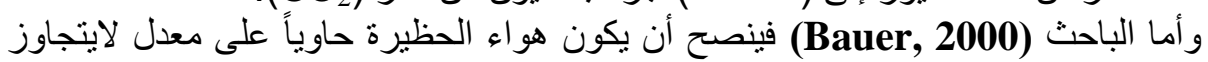
. $\mathrm{CO}_{2}$ (10)

وفي مجال آخر، قدم (Valentine,1964) تقريراً بأن زيادة الأمونيا في مساكن الدجاج الفاج

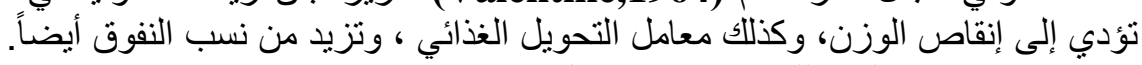

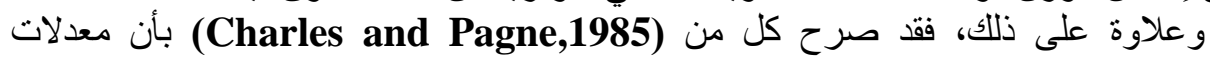

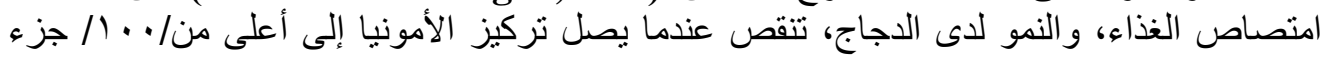

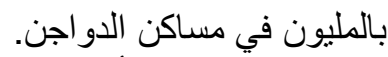
لقد درس تأثير الضوء في في زيادة إنتاجية الطيور الزراعية في أبحاث كثيرة وخاصة تلألك التي

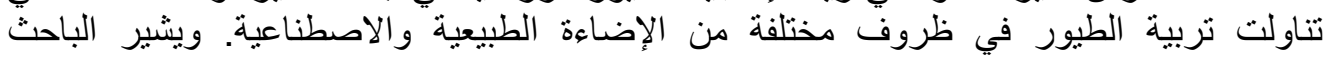

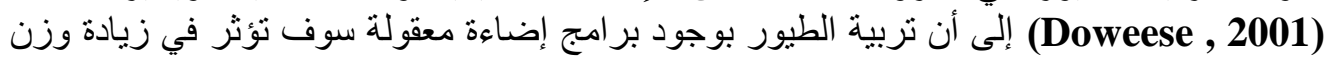
البيض ونوعية القشرة، ويتحسن بنفس الوقت إنتاج الدو اجن من البيض.

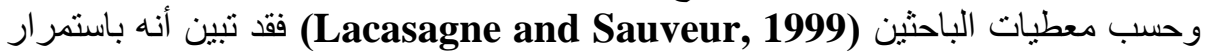

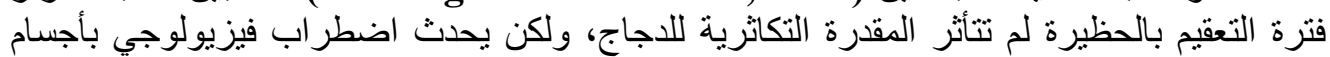

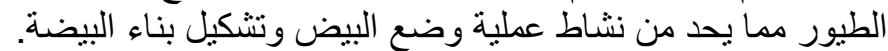

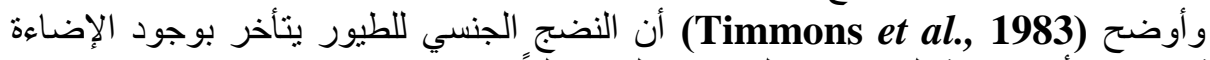

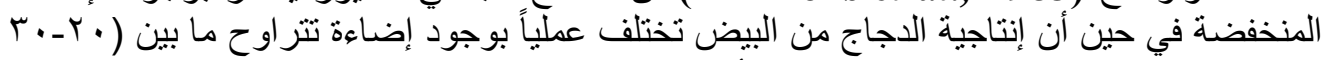

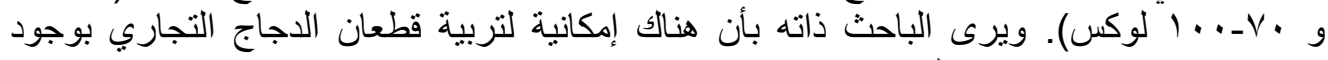

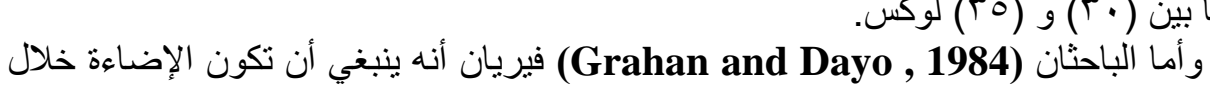

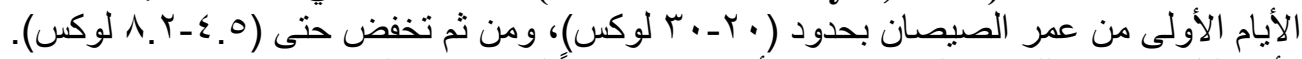

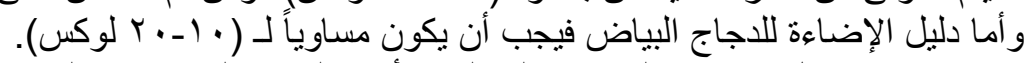

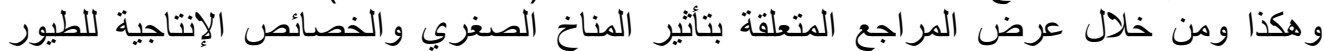

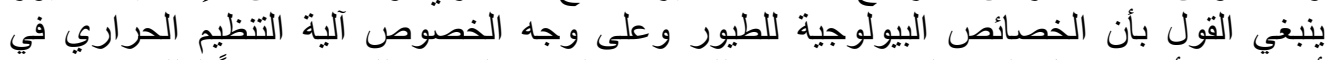

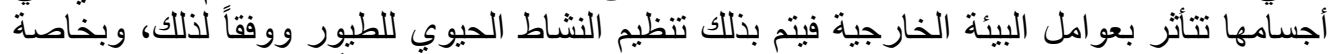

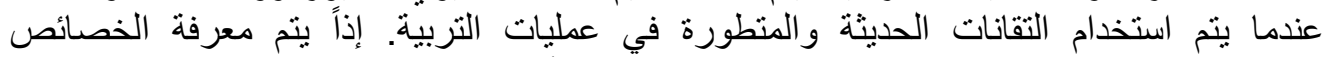

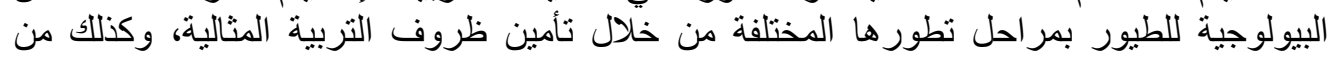

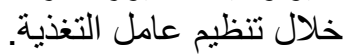

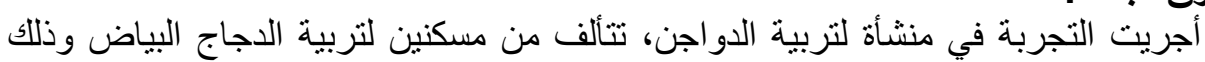

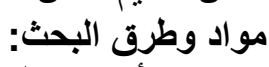

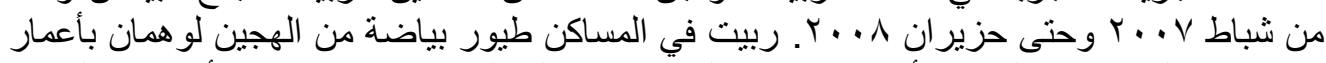

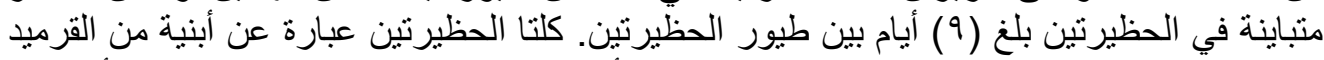

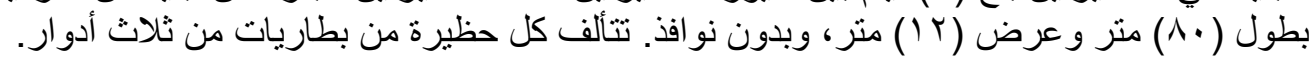

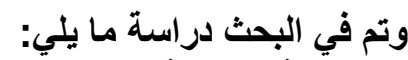

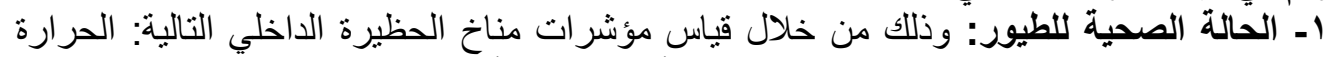

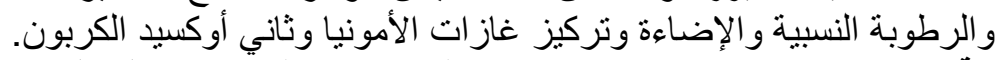

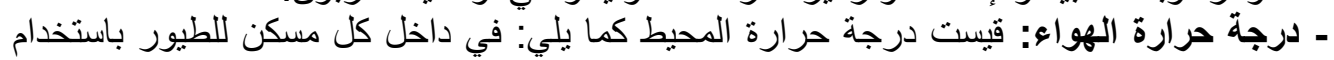
ميزان الحرارة العادي (المحرار).

Fayoum J. Agric. Res. \& Dev., Vol.23, No.2, (B) July, 2009 
ـ الرطوبة النسبية للهواء: قيست الرطوبة النسبية باستخدام المرطاب الثعري (مقياس الرطوبة

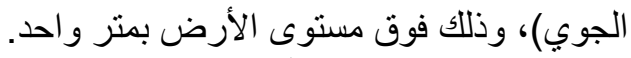

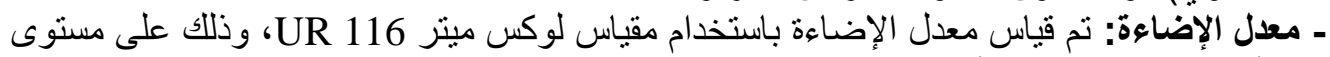

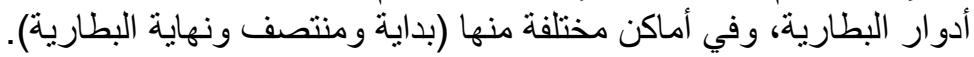

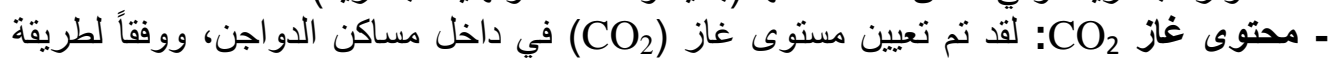
(Taylor, 1958)

- محتوى غاز الأمونيا (النشادر): استخدم لتحديد محتوى الأمونيا الطريقة التي تنصح بها جمعية (AOAC)

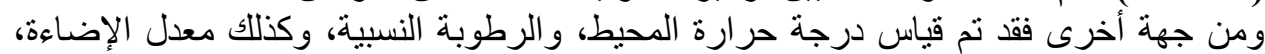
وتم تحديد محتوى غاز ات فرى في

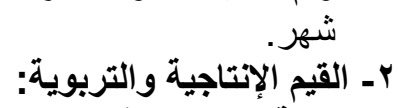

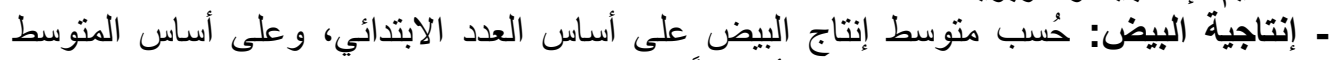

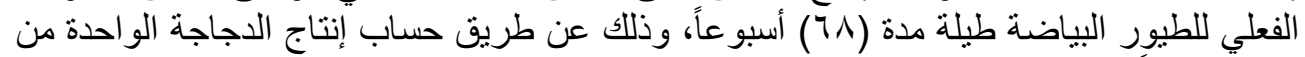
البيض يومياً.

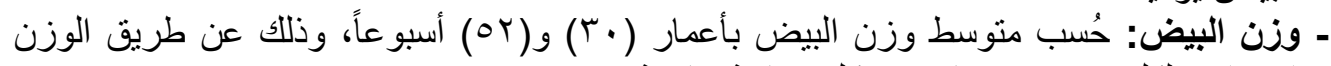

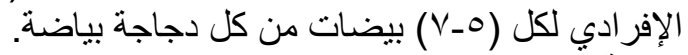

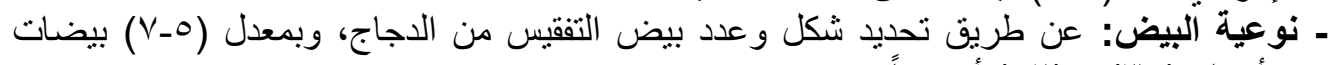

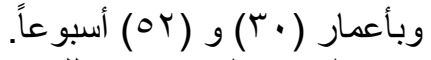
- عمر النضج الجنسي: وذلك عن طريق حساب ألكاريخ (موعد) وضع أول بيضة من كل دجاجة منفردة. أعدت المعطيات الناتجة باستخدام جدول سيتيودنت لعينتين مستقلتين.

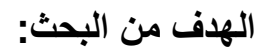
لقد كان الهذف من إجراء البحث هو دراسة تأثثير عوامل المناخ الداخلي المختلفة في المؤشرات الإنتاجية للاجاج البياض الباض من الهجين التجاري اءلي لو همان.

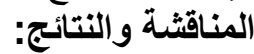

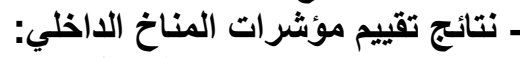

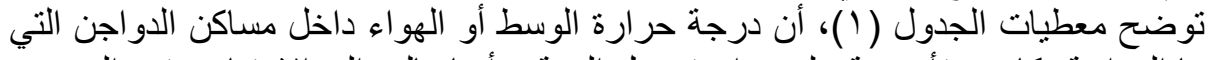

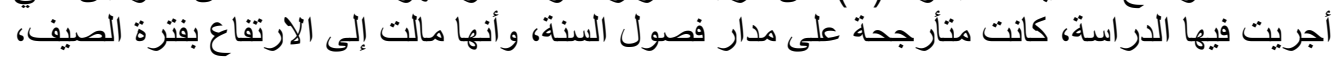

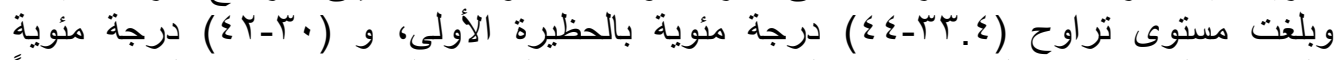

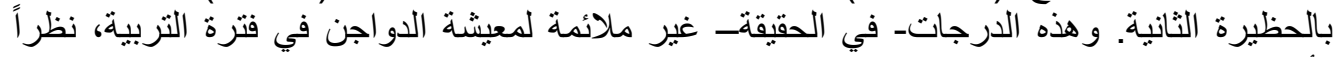

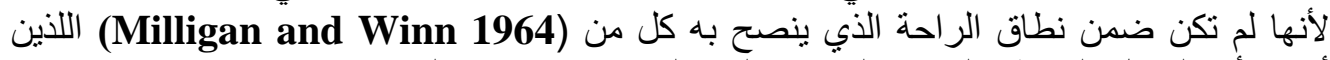

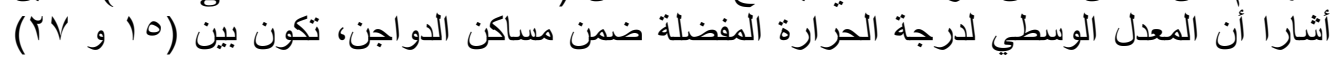

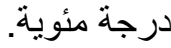

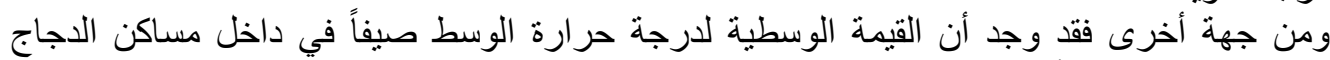

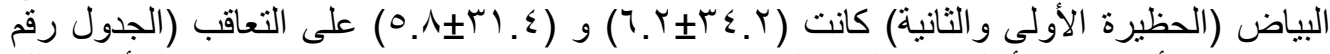

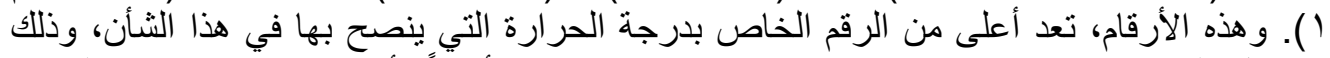

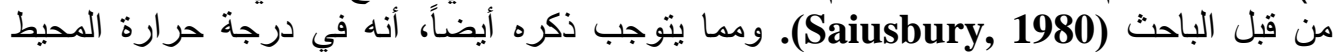

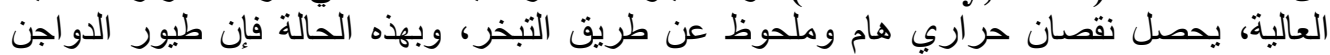

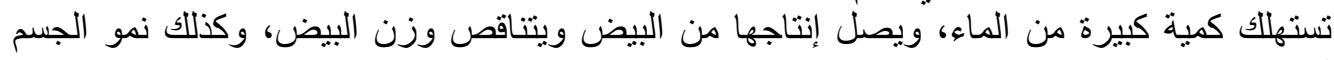

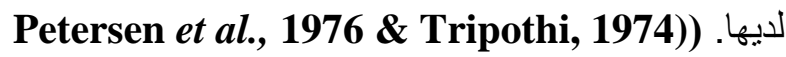

جدول رقم (1): القيم الوسطية لدرجة حرارة الهواء المئوية داخل مساكن الدجاج البياض حسب فصول السنة

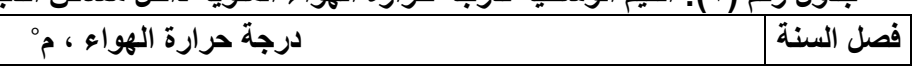

Fayoum J. Agric. Res. \& Dev., Vol.23, No.2, (B) July, 2009 


\begin{tabular}{|c|c|c|c|c|c|c|}
\hline \multicolumn{3}{|c|}{ الحظيرة الثاتية } & \multicolumn{3}{|c|}{ الحظيرة الأولى } & \\
\hline 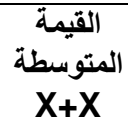 & $\begin{array}{c}\text { القغيمى } \\
\text { min }\end{array}$ & القيمة العظمى & القيمة المتوسطة & القيمة الصغرى & القيمة العظىى & \\
\hline 20.4 & 20.1 & 23.0 & 20.5 & 19.7 & 21.5 & الربيع \\
\hline 31.4 & 42.0 & 30.0 & 32.2 & 33.4 & 44.0 & الصيف \\
\hline 18.8 & 18.0 & 22.0 & 20.0 & 18.0 & 21.5 & الخريف \\
\hline 17.1 & 19.0 & 14.0 & 18.2 & 15.0 & 20.5 & الشتاء \\
\hline
\end{tabular}

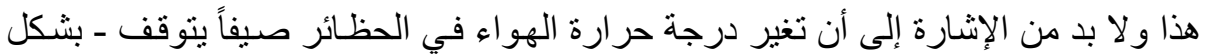

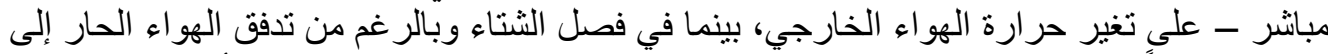

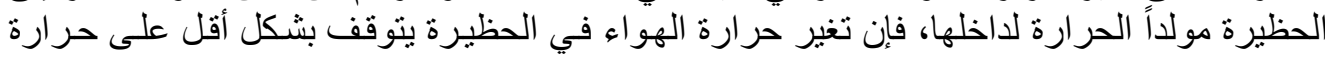

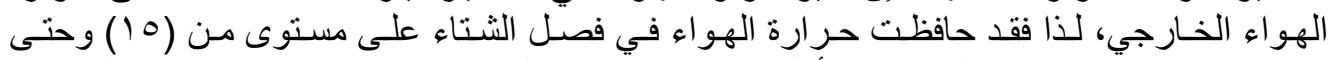

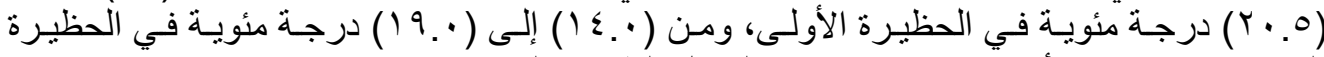

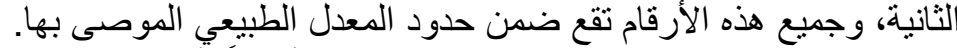

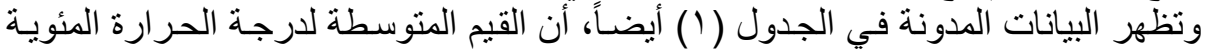

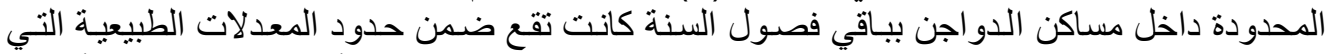
ينصح بها كل من الباحثين (Milligan and Sainisbury, 1974)، و أن هذه القيم كانت أفضل بالحظيرة الثانية وبجميع فصول اليانين السنة. وتبين نتائج البحث المدونـة في الجدول (r) القيم الوسطية للرطوبـة النسبية داخل مسـاكن الـجاج

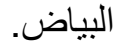

جدول رقم (ץ) القيم المتوسطة للرطوبة النسبية للهواء في مساكن الاجاج البياض بفصول السنة المختلفة (\%)

\begin{tabular}{|c|c|c|c|c|c|c|}
\hline \multicolumn{3}{|c|}{ الحظيرة الثانية } & \multicolumn{3}{|c|}{ الحظيرة الأولى } & \multirow[b]{2}{*}{ فصل السنة } \\
\hline $\begin{array}{c}\text { المتوسطة } \\
\text { X } \\
\text { X } \\
\end{array}$ & القيمة الصغرى & $\begin{array}{l}\text { القظيمى } \\
\text { max } \\
\end{array}$ & $\begin{array}{c}\text { المتوسطة } \\
\text { X } \\
\text { X } \\
\end{array}$ & القيمة الصغرى & القيمة العظمى & \\
\hline 71.3 & 69.0 & 75.0 & 70.0 & 69.3 & 79.0 & الربيع \\
\hline 62.0 & 60.0 & 72.0 & 68.7 & 57.0 & 70.7 & الصيف \\
\hline 75.0 & 72.0 & 78.0 & 74.4 & 71.0 & 78.0 & الخريف \\
\hline 77.0 & 69.0 & 78.0 & 75.0 & 70.0 & 76.0 & الثتاء \\
\hline
\end{tabular}

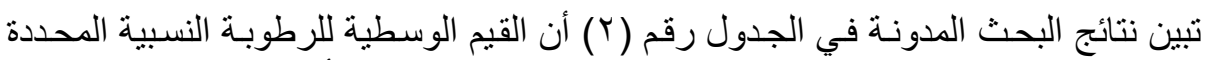

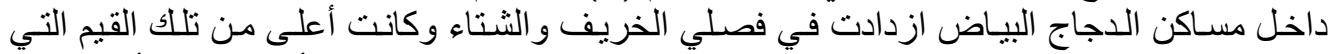

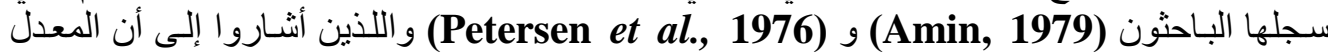

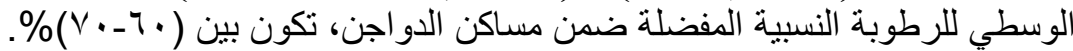

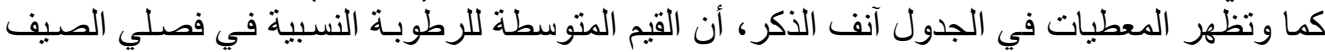

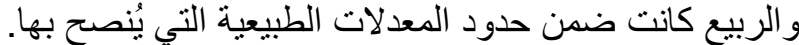

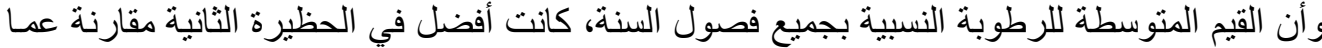
جدول رقم (ץّ) معدل الإضاءة في مساكن الاجاج البياض ، لوكس الإولى

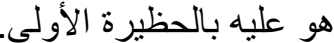

\begin{tabular}{|c|c|c|c|c|c|c|}
\hline \multicolumn{6}{|c|}{ الإضاءة ، لوكس } & \multirow{3}{*}{ فصل السنة } \\
\hline \multicolumn{3}{|c|}{ الحظيرة الثانية } & \multicolumn{3}{|c|}{ الحظيرة الأولى } & \\
\hline البطارية & منتصفية & بداية البطارية & نهاية البطارية & منتصفية & بلبارية & \\
\hline 30.0 & 28.0 & 27.0 & 25.0 & 24.0 & 22.5 & الربيع \\
\hline
\end{tabular}

Fayoum J. Agric. Res. \& Dev., Vol.23, No.2, (B) July, 2009 


\begin{tabular}{|c|c|c|c|c|c|c|}
\hline 32 & 30.7 & 28.5 & 28.5 & 26.0 & 24.7 & الصيف \\
\hline 30.5 & 29.0 & 27.0 & 27.2 & 24.5 & 26.3 & الخريف \\
\hline 30.0 & 28.0 & 26.5 & 27.9 & 24.5 & 25.0 & الثتاء \\
\hline
\end{tabular}

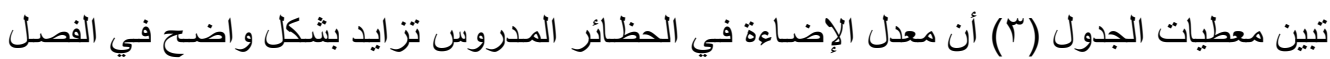

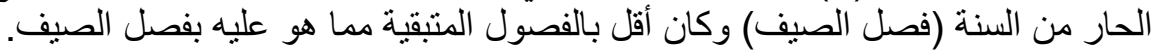

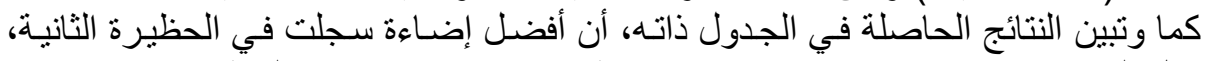

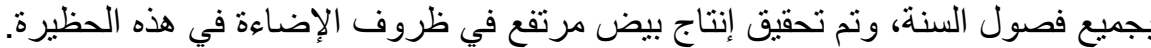

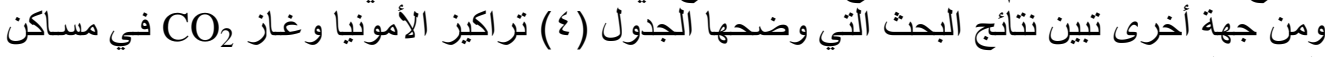

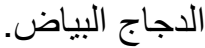

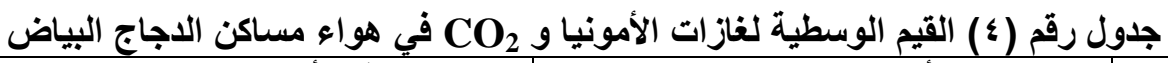

\begin{tabular}{|c|c|c|c|c|c|c|}
\hline \multicolumn{3}{|c|}{ ثاني أوكسيد الكربون (\%) } & \multicolumn{3}{|c|}{ الأمونيا، جزء بالمليون } & \multirow[b]{2}{*}{ الحظيرة } \\
\hline القيمة المتوسطة & $\begin{array}{c}\text { الصغرى } \\
\text { min }\end{array}$ & العظمى & $\begin{array}{c}\text { المتوسطة } \\
\text { X+X }\end{array}$ & $\begin{array}{c}\text { الصغرى } \\
\text { min } \\
\text { القيمة }\end{array}$ & القيمة العظمى & \\
\hline 0.16 & 0.05 & 0.09 & 8.5 & 6 & 12 & الأولى \\
\hline 0.15 & 0.04 & 0.07 & 8.2 & 7 & 11 & الثانية \\
\hline
\end{tabular}

وتنين نتائج البحث التي يوضحها الجدول (4) لمحتوى الأمونيا داخل مسـاكن الدجاج البياض أن القيم

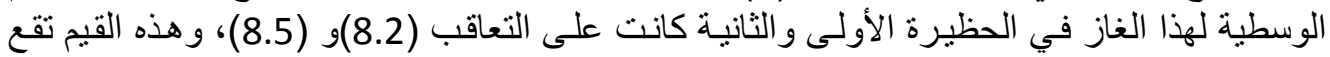

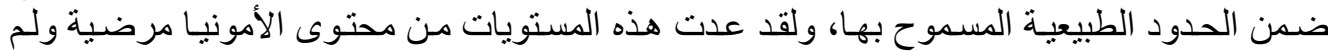

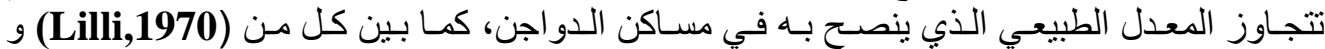

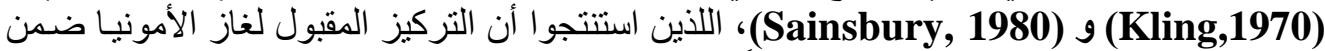

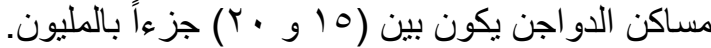

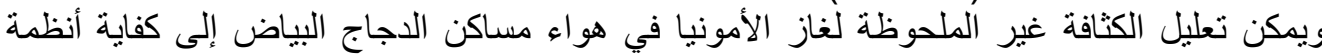

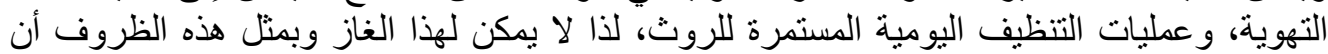

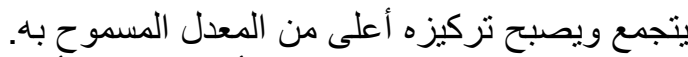

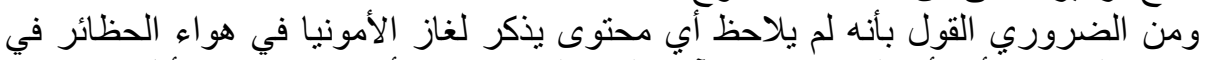

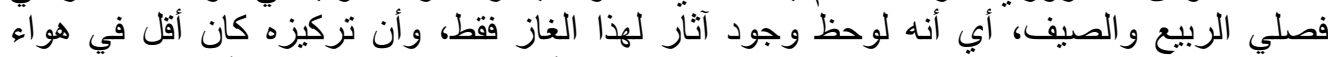

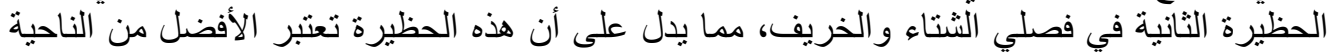
الصحية لتربية الطيور البياضة فئة البناء لقد عد الباحثون (Smith, 1974)، (Quarles and Kling, 1974) النسبة المئوية لغاز

الجي الهو اء الجوي لمساكن الدو اجن منسباً (مؤشراً) لتقييم كفاية التهوية في هذه المساكن. ويظهر الجدول رقم (0)، أن هذه النسبة من غاز

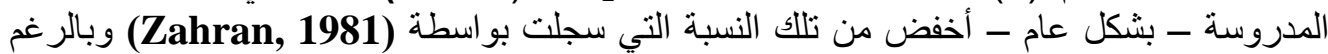

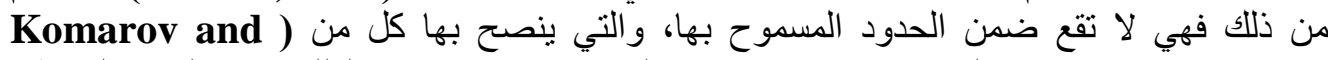

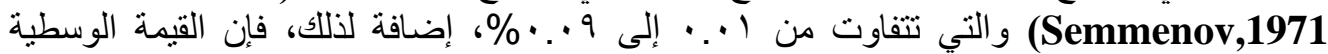

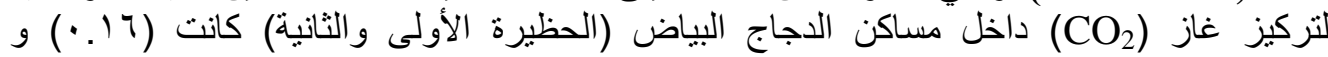

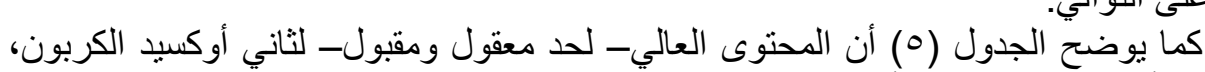

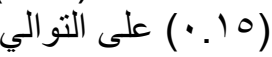

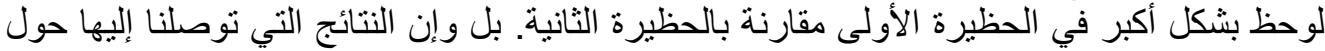

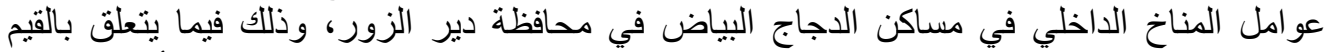

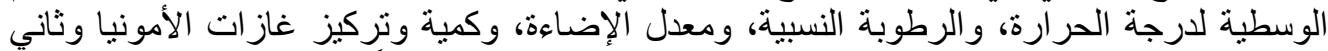

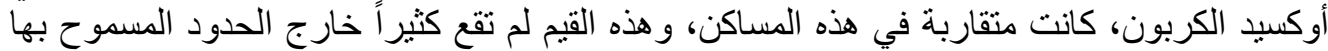

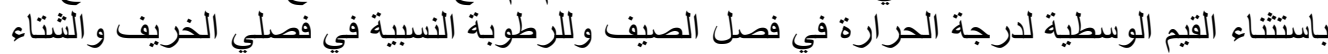

Fayoum J. Agric. Res. \& Dev., Vol.23, No.2, (B) July, 2009 
فقد كانت خارج الحدود المسموح بها، بل وأعلى من القيم التي ينصح بها البها الباحثون في مجال تربية

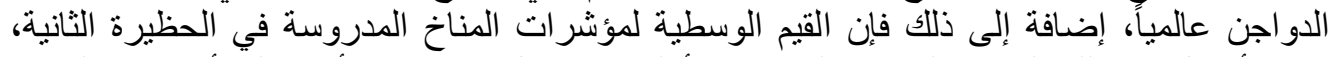

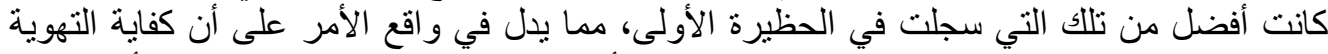

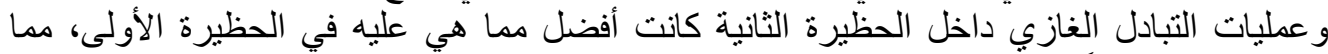

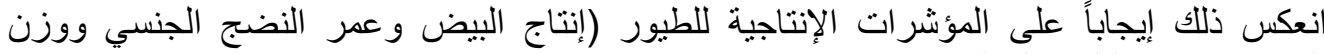
البيض و عدد ودليل شكل البيض).

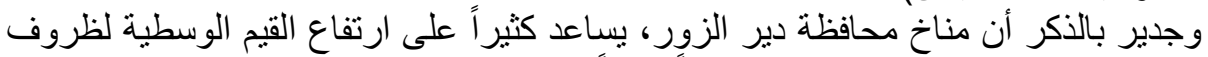

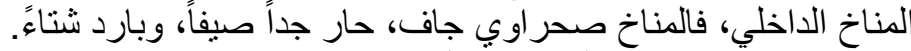
نتائج المؤشرات الإنتاجية والتربوية:

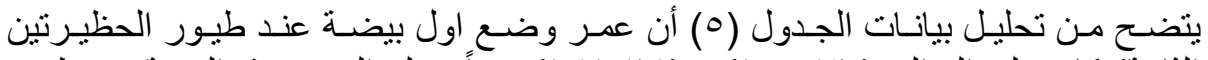

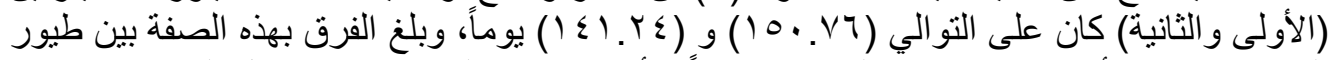

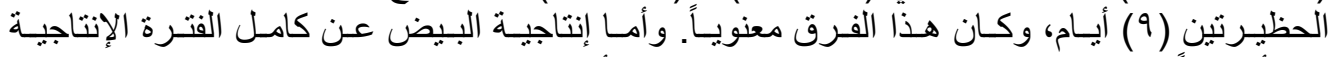

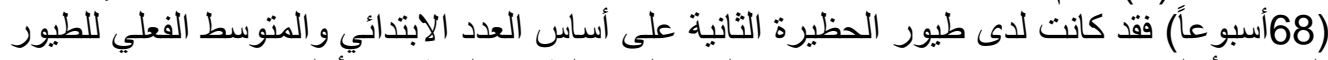

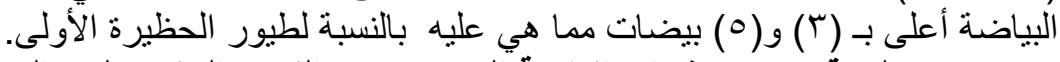

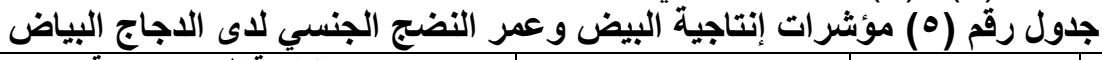

\begin{tabular}{|c|c|c|c|c|}
\hline ع ع على أساس & عن عمر (آنَّة & عمر النضج & & ت \\
\hline اللطيور البياضةً & للطيور البياضئة & (أيام) & البياض(دجاجة) & الحهيز \\
\hline $0 . \overline{0.80 \pm 234.95}$ & $\begin{array}{c}1.26 \pm 217.28 \\
\end{array}$ & $\begin{array}{c}0.29 \pm 150.76 \\
\end{array}$ & $\begin{array}{l}1785 \\
1509\end{array}$ & الأولى \\
\hline $1 . \overline{ \pm 239.15}$ & $1.57 \pm 220.52$ & $\begin{array}{c}0.43 \pm 141.24 \\
\end{array}$ & $\begin{array}{c}1091 \\
945\end{array}$ & الثانية \\
\hline
\end{tabular}

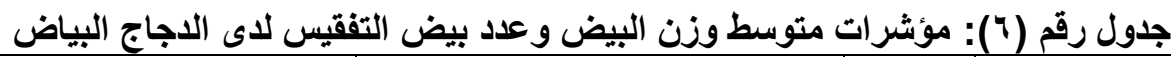

\begin{tabular}{|c|c|c|c|c|c|}
\hline \multicolumn{2}{|c|}{ عدد بيض التفقيس (\%) بأعمار } & \multicolumn{2}{|c|}{ وزن البيض (غ) بأعمار } & \multirow{2}{*}{ الطيور } & \multirow{2}{*}{ 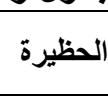 } \\
\hline (52) أسبوع & (30) أسبوع & (52) أسبوع & (30) أسبوع & & \\
\hline$\overline{\bar{T}}$ & $\overline{\bar{T}} \overline{\bar{C}}$ & $\begin{array}{c}0.13 \pm 61.53 \\
-\end{array}$ & $\begin{array}{c}0.109 \pm 54.47 \\
- \\
-\end{array}$ & $\begin{array}{c}1129 \\
805 \\
1698 \\
1397\end{array}$ & 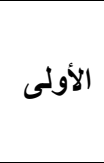 \\
\hline$\overline{\bar{T}}$ & 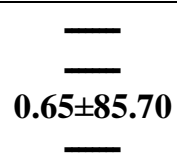 & $\begin{array}{c}\overline{0^{2}} \\
- \\
-\end{array}$ & $\begin{array}{c}0.10 \pm 53.29 \\
- \\
-\end{array}$ & $\begin{array}{c}1007 \\
791 \\
1051 \\
883\end{array}$ & الثانية \\
\hline
\end{tabular}

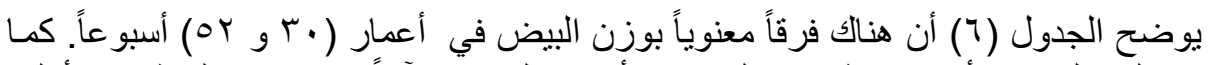

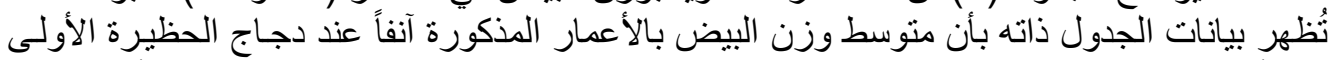

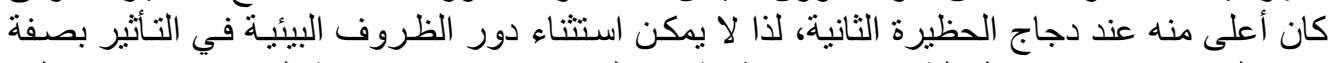

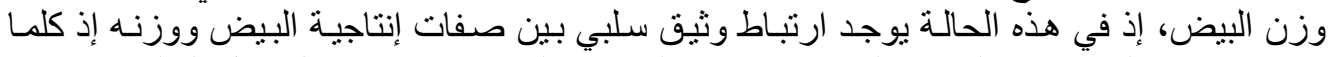

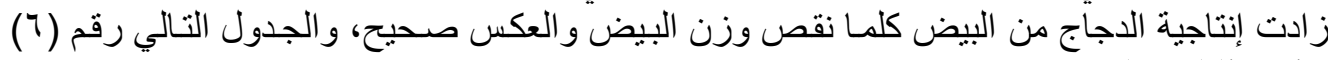

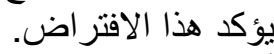
أما بالنسبة لصفة عدالفد بيض التفريخ فقد وجد بأن هناك فرقاً بهذه الصفة بين طيور الحظيرنين، وكان هذا الفرق غير معنوي (أنظر الجدول آ).

Fayoum J. Agric. Res. \& Dev., Vol.23, No.2, (B) July, 2009 


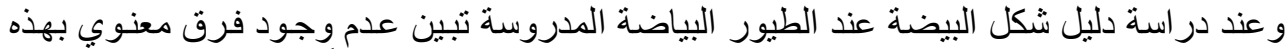

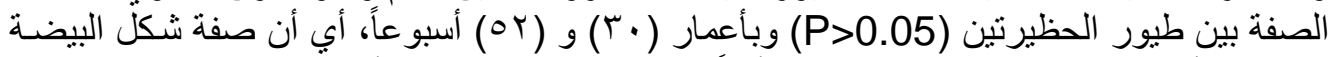

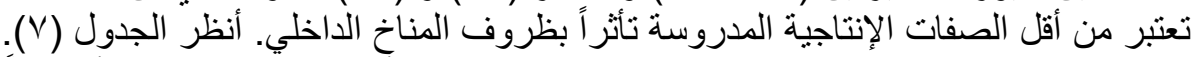

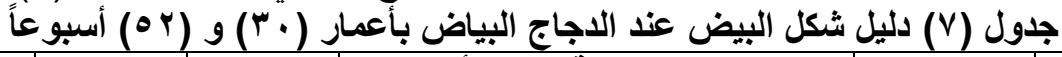

\begin{tabular}{|c|c|c|c|c|c|c|}
\hline \multirow{2}{*}{$\begin{array}{c}\text { T } \\
\text { t.d } \\
\text { t.d }\end{array}$} & \multirow[b]{2}{*}{ C.V } & \multirow[b]{2}{*}{7} & \multicolumn{2}{|c|}{ دليل شكل البيضة (\%) بأعمار } & \multirow{2}{*}{ (لاجاجة) } & \multirow[b]{2}{*}{ الحظيرة } \\
\hline & & & 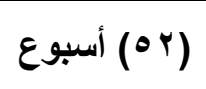 & ( · إ) أسبوع & & \\
\hline $\begin{array}{l}1.06 \\
1.35\end{array}$ & $\begin{array}{l}2.97 \\
3.81\end{array}$ & $\begin{array}{l}2.13 \\
2.74\end{array}$ & $0.18 \pm 72.14$ & $\begin{array}{c}0.28 \pm 71.89 \\
-\end{array}$ & $\begin{array}{l}1600 \\
1500\end{array}$ & الأولى \\
\hline $\begin{array}{l}1.06 \\
1.35\end{array}$ & $\begin{array}{l}3.20 \\
3.90\end{array}$ & $\begin{array}{l}2.29 \\
2.84\end{array}$ & $0 . \overline{0.09 \pm 72.95}$ & $0.07 \pm 71.38$ & $\begin{array}{c}1050 \\
950\end{array}$ & الثانية \\
\hline
\end{tabular}
تظهر نتائج البحث أن طيور الحظيرة الثانية بلغت عمر النضج الجنسي أبكر بمعدل (9) أيام، مقارنـة • ز اد إنتاج البيض الحظيرة الاولى. أساس منوسط الطيور البياضة عن كامل الفترة الإنتاجية (1/أسبو عاً) مقدار (0) بيضات مقارنة بطيور الحظيرة الأولى البطي.

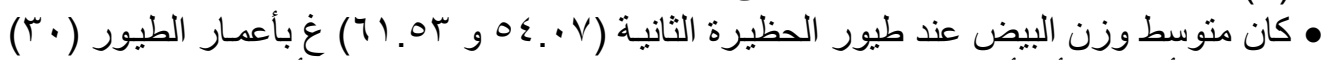

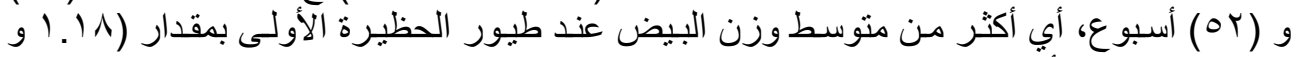

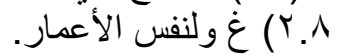

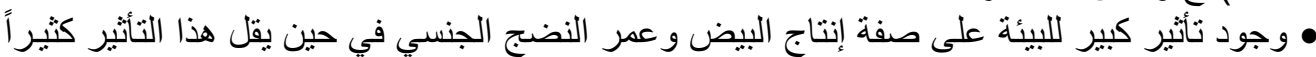

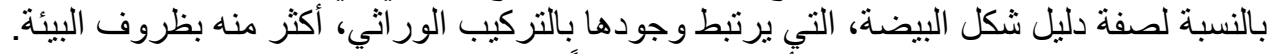

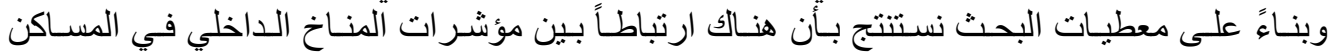

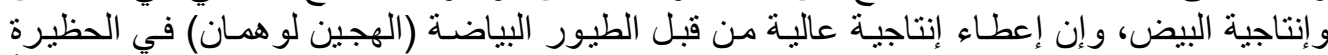

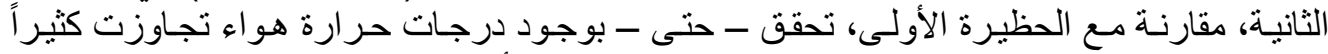

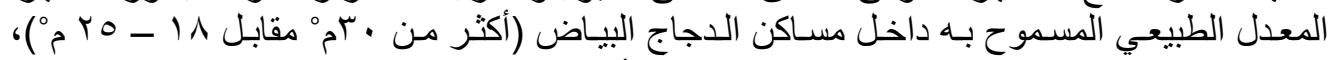

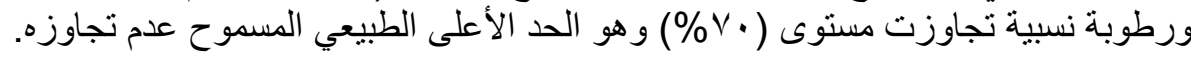

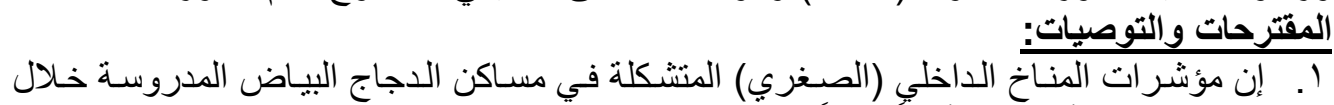

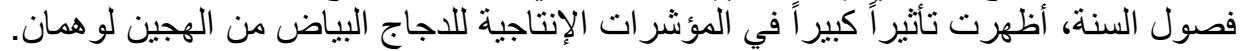

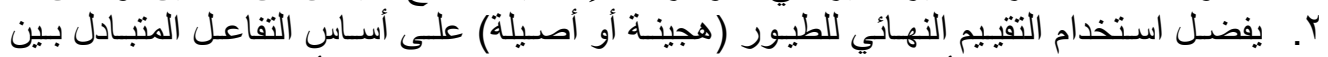

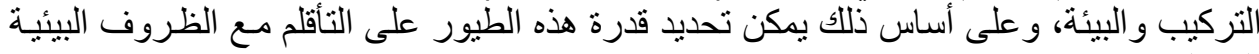
المختلفة.

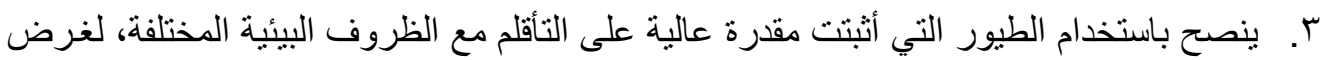

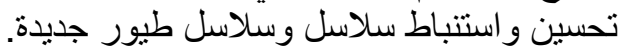

REFERENCES المراجـع

Airmolaifa, A.L. 1989. Which tempteture is better in the houses of Poultry. J. Poultry . Moscow., No. $7: 33-42$.

Al-banki, A.I. and Al-Bashan, M.M. 2008. Studies on efficiency of ventilation in boilers and layers 'hens at Deir Ezzour governorate with special reference to campylobacteriosis. J. of Damascus Univ. in Agric. Researches (under publication).

Fayoum J. Agric. Res. \& Dev., Vol.23, No.2, (B) July, 2009 
Amin , f . 1979. Studies on the sanitary condition of poultry in upper Egypt , ph. D. Thesis Fac. Vet . Mel . Assiut Univ.

Anigof, M.K. 1974. The effect of microclimate on production and physiological case of laying hen's during breeding in battery cages and on the floor Ph.D. Thesis. Agricultural University of Kazan .77.

AOAC, 1975. Official methods of analysis, $12^{\text {th }}$ ED. published by the association of AOAC, P.O BOX Benjamin Franklin station.

Arora, R.G. and Karla, D.S. 1992. The effect of the factors of microclimate on production traits of laying hen's. Indian Journal of Animal Science . 43 (12) . $1095-1096$.

Asaj, A. Audi, S. and Paukovic, M. 1970. Unweltverhaltniss bei Badehaltung Von Legenuhnern . Deutsch, Tierazt 1. Wschr., 77 : 166 - 169 .

Bauer John, D. 2000. the experiments of adaptation of laying hen's for conditions of breeding in battery cages and on the floor. Aust .J. Agric. Res., 19: 283 - 294.

Calmicof, M.F. and Alepastrof, A.P. 1975. Idial microclimate in houses of poultry. Vol. Sci. Works. Agri. Research . institute . Saratof : $75-86$.

Charles, D. and Payne, C. 1985. The influence of graded level of atmospheric ammonia on chickens . Effect of respiration on per for mance of broiler replacement growing stock . Brit . poultry Sci , 7:177184 .

Doweese, W.P. 2001. The effect of different programs of lighting on growth and production of poultry. J. Anim. Sci., 32: 394-397.

Dutt, R.H. 1998. The microclimate and the production during keeping birds on the floor. Brit. Poultry. Sci., 6: 166-174.

Grahan, E.F., Dayo, C.M. 1984. The of the lighting programs for increasing of production of laying hen's. the proceeding of the $14^{\text {th }}$ technical conference on poultry. Colombia. Missouri.

Jack, M. and Blum, L. 1978. The influence of high constant environmental temperature and energy lenel in the diet on the performance of laying hen. Arch, Gefungel, 42: 216 - 220.

Kling, H.F. 1970. Gas and particulate levels in poultry houses. M.S. Thesis. the Pennsylvania state Univ .

Komarov, N. and Semmenov, K. 1974. Influence of air ionization on broiler chocks. trudy Vsesoyuznogo Ins Eksperimentalnoi Veterinorii. 39: 362-374.

Lacasagn, L. and Sauveur, B.N. 1999. Yctyhemeres de 26 eta 28 heures et depot de la coguille chez. la poule dometique. annalas de zootechnic. Vol. 22. No.1: 103-199.

Lillie, R. 1970. Air pollutants affecting the performance of domestic animals Rev. Agric. Handbook No-380 -U.S, Dep. of Agric.

Fayoum J. Agric. Res. \& Dev., Vol.23, No.2, (B) July, 2009 
Milligan, J. and Winn, L. 1974. The in fluence of and temperature humidi ty broiler performance in environmental on chamber- Poultry Sci . 43:817 - 824.

Mou, L.J. and Katle, J. 1990. Effect of different environments and selection for persistency in laying hen's. Egg performance in laying hen's in two different environments (cages and floor). Norw. J. Agric. Sci. Vol. 4. 4 (3): 205-210.

Motis, E. and Danilofa, A.K. 1986. Microclimate in animal farms. Annals. Agric. Sci., Moscow Academy. 4:70-73 (in Russian).

Petersen, J. Chima, M. and Harst, P. 1976. Body temperature as am easure of acclimatization in laying hens. Zeitschrift fur Tierzuchtung und Zuchtangsbiolo -gie, 39(3-4) :237 - 35).

Reece, F. Lott, B. 1980. Effect of carbon dioxide on broiler chichen performance Poultry Sci ; 59:2400-2402.

Robinson, T.J. 2008. The increasing of the quantity of poultry production, nutritional and hatching. Proc. Aust. Soc. Anim. Prod. No. 8: 47-49.

Quarles, C. and Kling, H. 1974. Evaluation of ammonia and in factious bronchitis Vaccination stress and broiler performance and carcass quality. Poultry Sci. - 53:1592-1598.

Sainsbury, M. 1980. Poultry health and management . First Ed . published by Granad uplishing limited .

Samaha, H. 1991. Efficiency of Ventilation existing in some poultry houses at Behera and Alexandria Governorates. Assiut Vet. med. J.; Vol.24.

Smith, A. 1974. Changes in the average of weight and shell thickness of eggs produced by hen's exposed to high environmental temperatures. Tropical animal health and production. 6 (4): 240-244.

Taylor, W. 1958. The examination of water and water supplies. Seventh Ed . Thresh, Beale. and suckling .

Timmons, M.B. Stopes, T.D., Martin, G. 1983. Dark out housing, lighting programs providing result for broiler breeders. poultry. Digest. Vol. 43. No. 496: 286-289.

Tripothi, N. 1974. Studies on layers reared at higher environmental temperatures. Indian J. of. Animal Science., 46 (5): 253-259 .

Valentine, H. 1964. A study of the effect of a different ventilation rats on the ammonia concentration in atmosphere of broiler houses. Brit. Poultry. Sci., 5: 149-159.

\title{
THE EFFECT OF MICROCLIMATE FACTORS ON SOME PRODUCTIVE PARAMETERS FOR LAYING HEN'S
}

\author{
Al-Banki, A. I. \\ Department of Animal Production, College of Agriculture, University of \\ Alfurat, Deir Ezzour, Syria
}

Fayoum J. Agric. Res. \& Dev., Vol.23, No.2, (B) July, 2009 


\begin{abstract}
The research aims to study the effect of different climatic factors (temperature, relative humidity, intensity of gases- $\mathrm{NH}_{3} \& \mathrm{SO}_{2}$ ) in layers hens houses, on the productive parameters for Hybird (Lohman). The research was carried out in a house for breeding layers parents in Deir Ezzour governorate in Syria from February 2007 to June 2008. The results of the research showed that the studied microclimatic parameters in houses of layers during the seasons of the year, a great effect in the productive characters, was reflected positively on the performance (production and quality) characters of layer birds, in spite of that the mean values surpassed the allowed limits as temperature in summer, which was over (30) $\mathrm{C}$ compared to the natural average $(15-27) \mathrm{C}$, and the relative humidity in autumn and winter which reached a higher level than $(60-70) \%$. Also the results of the research show that the quality of producing eggs is considered on the productive characters which is influenced greatly by microclimatic conditions, where as we find that the index of egg's shape is considered the least traits influenced by environment, and it's linked greater with the genotype than microclimate. While the sexual maturity and the eggs weight are influenced by a strong link between genotype - environment.
\end{abstract}

Key words: Microclimate, Productive Parameters, Layers.

Fayoum J. Agric. Res. \& Dev., Vol.23, No.2, (B) July, 2009 